Research Article

\title{
Characteristic Parameter Optimization of Wireless Flaw Sensing System Based on Active Interference Suppression Algorithm
}

\author{
Wenping Wu \\ Information Engineering Department, Suzhou University, Suzhou 234000, China \\ Correspondence should be addressed to Wenping Wu; wuwenping251@ahszu.edu.cn
}

Received 2 November 2021; Revised 21 November 2021; Accepted 25 November 2021; Published 7 December 2021

Academic Editor: Gengxin Sun

Copyright (c) 2021 Wenping Wu. This is an open access article distributed under the Creative Commons Attribution License, which permits unrestricted use, distribution, and reproduction in any medium, provided the original work is properly cited.

\begin{abstract}
Based on the active interference suppression algorithm, this study combines the radar working mode and the interference type and realizes the effective detection of the flaw detection signal by successively processing the radar receiving signal and the filtering processing. Firstly, this article builds a simulation platform similar to the actual situation to verify the existing conventional active interference suppression algorithms. Secondly, for the detection of chirp active deception jamming signals entering from the main lobe, a radar active deception jamming detection method based on the characteristic parameter matching of the harmonic components of active deception jamming signals is proposed. After that, the spectral characteristics of the harmonic components of the deception interference signal are analyzed, and the center frequency and the tuning frequency of the real target echo are obtained. Finally, by establishing a frequency modulation parameter library for possible interference harmonic signal components, the acquisition phase of the radar gate by the jammer matched analysis with the preestablished frequency modulation parameter library is implemented to achieve active deception interference detection. This method can effectively detect active deception jamming signals in a complex tunnel environment. The interference suppression algorithms verified by simulation include noise FM interference suppression algorithm based on cancellation and distance false target interference suppression algorithm based on LFM radar summary processing. Through actual measurement data processing and analysis, the effectiveness of the method is verified and the idea of interference suppression is expanded. The construction of the simulation platform is obtained by appropriately modifying the actual parameters, a certain type of suppression jammer, and a certain type of deception jammer used in a certain countermeasure field test at a radar station.
\end{abstract}

\section{Introduction}

With the rapid development of microelectronics technology, the radar interference and interference countermeasures are becoming more and more diverse, and the processing is more complicated [1]. The most common passive interference is chaff interference, which can be filtered out by AMTI; in active interference, the common way of covering interference is noise interference. Generally, high-power jammers are used to generate noise signals to make the target submerged in noise. Among them, there are more forms of deceptive interference [2-5]. The interference can be performed separately from the parameters of the distance, speed, and angle, or multiple target parameters can be used for simultaneous interference. Active suppression interference is an important means of electronic attack, mainly by emitting high-power noise or noise-like waveforms. Covering the target echo, destroying or obstructing the detection and parameter estimation of the target by radar is widely used in foreign jamming systems [6-8]. From the EA-6B "Prowler" to the EA-18G "Growler", to the next-generation jammer (NGJ), active suppression interference has always existed as a basic interference method. As a substitute for the "Prowler," the "Growler" is mainly responsible for longrange support jamming (SOJ) and team jamming (ESJ) tasks. The AN/ALQ-99 tactical jamming pod has been widely used and improved many times by the "Prowler" and has been installed on the "Growler." It mainly performs electronic suppression tasks. Its modes of action include targeted jamming, dual-frequency jamming, and sweep frequency interference, which can effectively interfere with warning radars and ground guidance tracking radars [9-11]. 
Feng [12] established a unified model for suppressing interference. By analyzing the interference power spectrum density, the two characteristics of coherence time and coherence bandwidth were introduced, and finally, fast interference and slow interference, frequency blocking interference, and frequency selective interference were effectively separated. In this method, the power spectrum estimation of time-varying interference is the key, and the classification of locally unstable interference is still to be resolved. Orr [13] modeled interference and echo based on the signal cone theory and discussed the method of detecting and identifying real target echo and deceptive interference signal. However, this method is limited to phase quantization, only when the number of quantization bits is low. It is only effective under the next. Chiariotti [14] uses a joint filtering algorithm of EMD and fractional Fourier transform (FrFT) to suppress suppressing interference components in chirp signals, effectively reducing the impact of interference on echo. Fayyad [15] is aimed at high-power interference in the case of tracking information distortion, and an EMDbased monopulse radar processor structure is proposed. Zekavat [16] uses fast wavelet transform (FWT) to suppress interference and detect targets. Other scholars analyzed the performance of search radar and tracking radar under blocking jamming, deduced the probability distribution of target detection in jamming environment, and studied the change in interference-to-noise ratio in the radar receiver in the presence of multiple dynamic noise jammers [17-21]. Aiming at the problem of the performance degradation of target tracking in the interference zero zone in the traditional method, the researchers studied the countermeasures for air-to-air single-target tracking in the jamming environment, which improved the stability and continuity of single-target tracking. Some scholars use the maximumlikelihood estimation (MLE) technology to determine the target angle of arrival (DOA) under main lobe interference, study the adaptive main lobe interference suppression technology for monopulse radar, and propose a digital beamforming structure and processing algorithm. One main lobe interference and multiple side lobe interferences are reset to zero to ensure the accuracy of the monopulse radar's estimation of the target DOA [22].

To better protect the performance of own radar, we must first study the characteristics of various interference signals. This article analyzes common interference signals from both passive interference and active interference. In radar electronic warfare, active suppression of jamming plays an important role. Radar active suppression jamming masks and suppresses the true target echo by radiating high-power noise or noise-like interference signals, resulting in the radar not being able to effectively obtain target information. In this regard, this article carried out the research on anti-active suppression interference based on the idea of interference detection and identification of interference suppression. This study analyzes the aiming jamming, blocking jamming, sweeping jamming, and comb spectrum jamming models based on noise FM signals and discusses the characteristic performance of different types of jamming after acting on the radar receiver. Based on the proposed characteristic parameters, the effective detection of interference is realized, and the decision tree method is used to realize the classification and identification of different types of active suppression interference, which achieves a higher probability of interference detection and correct identification. Through the processing and analysis of the measured data, the validity of the proposed feature parameters and the feasibility of the identification method are verified.

\section{Construction of a Tunnel Radar Detection Sensor Model Based on Active Interference Suppression Algorithm}

2.1. Active Interference Suppression Algorithm Topology. The suppression of active suppression interference is currently mainly carried out from the aspects of signal, information, and radar systems. The signal level mainly uses the difference between the useful signal and the interference signal and uses the corresponding algorithm to suppress [23]. Figure 1 is the structure of the active interference suppression algorithm. The information level is anti-interference in the field of radar data, such as the use of information fusion technology to anti-interference.

There are many classification methods for radar active suppression interference. According to the generation mechanism, that is, the different modulation methods of the interference signal, the interference can be divided into radio frequency noise interference, noise frequency modulation interference, and noise amplitude modulation interference. According to the type of active suppression interference on the radar receiver, it can be divided into blocking interference. In addition, according to the spatial position relationship of radar, target, and jammer, radar active suppression interference can be divided into support interference and SSJ. Among them, support interference can be divided into SOJ, ESJ, and close-range jamming (SFJ).

$$
u(x, t)=a(x, t)+b(x, t)+c(x, t) .
$$

The pulse compression technology uses a large-timewidth pulse signal. After receiving the echo signal, the largetime-width pulse needs to be compressed into a narrow pulse signal at the beginning of signal processing to increase the range resolution. Radar signal processing is implemented in the digital domain. The essence of pulse compression technology is matched filtering, which is called matched filtering in the analog domain and pulse compression in the digital domain [24].

$$
\begin{aligned}
C R & =\left(\frac{\mathrm{CAR}}{\mathrm{CLC}}\right) \times 100 \%, \\
\mathrm{RTA} & =\frac{(T P+I E)}{T A} \times 100 \% .
\end{aligned}
$$

According to the design theory of matched filter, it can be known that the phase of the designed pulse compression filter should be conjugated to the signal phase. The pulse compression technology is divided into two processing methods: time domain and frequency domain. Generally, it 


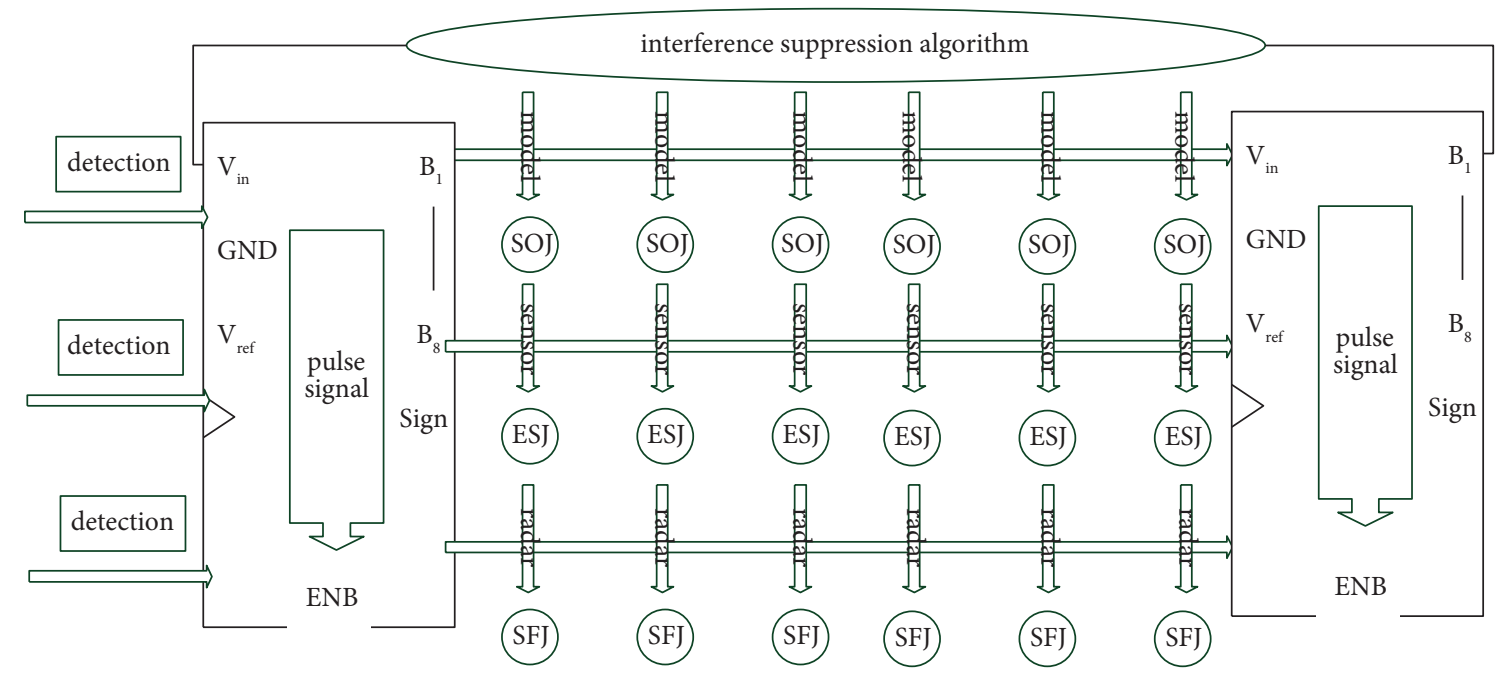

FIgURe 1: Active interference suppression algorithm structure.

is processed in the time domain when a smaller pulse pressure ratio is required and in the frequency domain when a large pulse pressure ratio is required.

$$
G(x)= \begin{cases}g(x) \times[0, x, 1], & x \subset X, \\ g(x) \times[x, 1,0], & x \subset Y, \\ g(x) \times[1,0, x], & x \subset Z .\end{cases}
$$

Although they are all linear frequency modulation broadband interference signals, due to the different frequency modulation of the two broadband interference signals, it is difficult to make two or more linear frequency modulation broadband interference signals with different modulation frequencies under the same window function and window length.

$$
\begin{aligned}
c & =\operatorname{Pr}(c \mid o) \times \operatorname{Pr}(o) \times I o U=\operatorname{Pr}(c) \times I o u, \\
g(m, t) & =\frac{n \times(h(m, t) / k(i, t))}{\sum_{i=1}^{n} h(i, t) / k(i, t)} .
\end{aligned}
$$

The same problem exists for sine FM broadband interference. Since the modulation frequency of a sine FM signal changes with time, the same window function and window length will have certain time-frequency two-dimensional characteristics at different moments. The difference results in a better time-frequency two-dimensional aggregation characteristic of its frequency spectrum in certain time periods.

2.2. Radar Flaw Detection Processing Flow. Generally, the error detection processing requirements for radar are small enough to ensure that the interference frequency covers the working frequency band of the radar. Therefore, the interference signal needs to be accurately guided, which places high requirements on the electronic support means (ESM) of the interferer. Targeted interference has a high-power utilization rate and can ensure sufficient interference energy to enter the radar receiver, which is the most commonly used method to suppress interference. However, modern radars generally use anti-jamming methods such as frequency agility, which puts forward higher requirements on the timeliness and accuracy of frequency guidance for targeted jamming. For a pulse compression radar with a pulse compression coefficient of $D$, after the echo passes through the compression network, the peak power becomes $D$ times the original. Therefore, when the received signal contains only echoes, the peak-to-average power ratio after pulse compression is usually larger. When there is interference in the received signal, the interference power is usually much greater than the echo power. After the received signal passes through the pulse compression network, the interference energy increases the side lobe of the echo pulse compression, and even the side lobe floods the main lobe, resulting in peak-to-average power. Table 1 is the radar detection processing parameters.

For MTD, the passband and stop band of each narrowband filter are located in different channels. Since each filter has side lobes, the size of the side lobe determines the clutter suppression ability, so the side lobe is generally reduced by windowing. In actual engineering, the implementation of MTD is generally to first realize the filter bank function through FFT and then add windows to reduce the side lobes. MTD technology often detects target signals together with clutter map technology. The clutter map is a quantitative description of the surrounding environment of the radar. In DRFM, the analog-to-digital conversion of the intercepted signal and the digital-to-analog conversion process of the modulated signal will cause the signal to produce harmonic distortion. The analog-to-digital conversion process and digital-to-analog conversion process in the project are realized through an analog-to-digital converter $(\mathrm{ADC})$ and a digital-to-analog converter (DAC).

The process of quantizer mapping the amplitude of the signal to discrete levels is a nonlinear process, which will inevitably lead to harmonic distortion of the output signal. Although increasing the number of quantization bits of $\mathrm{ADC}$ and $\mathrm{DAC}$ can help reduce the degree of harmonic 
TABLE 1: Radar flaw detection processing parameters.

\begin{tabular}{|c|c|c|c|}
\hline Index number & First-level indicator & Second-level indicator & Third-level indicator \\
\hline \multirow{3}{*}{1} & \multirow{3}{*}{ Signal capability } & Return on node & 0.11 \\
\hline & & Signal turnover rate & 0.09 \\
\hline & & Operating growth rate & 0.06 \\
\hline \multirow{3}{*}{2} & \multirow{3}{*}{ Signal service ability } & Signal chain distribution accuracy & 0.12 \\
\hline & & On-time rate of signal chain distribution & 0.11 \\
\hline & & Logistics service integration & 0.22 \\
\hline \multirow{2}{*}{3} & \multirow{2}{*}{ Information technology capabilities } & Information coverage & 0.14 \\
\hline & & Information degree & 0.32 \\
\hline
\end{tabular}

distortion, the number of quantization bits of $x$ bit will increase the sampling frequency $\mathrm{x} / 2$ times the storage capacity of the sampling frequency and a large amount of quantization bits than 1 bit. Because MTD uses a filter bank to filter out clutter, there is a complete target in each filter channel, and the clutter will be diluted, so MTD helps increase the signal-to-clutter ratio, but for targets flying along the tangential direction of the radar, this tangential target can be observed using the clutter map at the same time.

\subsection{Analysis of Signal Detection Process Factors. In the signal} detection process, each interference signal is independent and identically distributed, and the center frequency can be determined, or it can be a random variable subject to a certain distribution. The requirements for interference bandwidth can refer to blocking interference. According to the width of the sub-bands, comb spectrum interference can be divided into broadband comb spectrum interference and narrowband comb spectrum interference. In communication, single-frequency signals are often used as interference signals, which are called multitone interference, which is a kind of narrowband comb spectrum interference. This article mainly studies broadband comb spectrum interference. The notch of the secondary canceller is wider, and the stop band is widened, so the improvement factor is larger, and the filtering effect is better. But as the order increases, the passband of the canceller will also become fluctuating and not flat enough. This will affect the amplitude of the target signal after passing through the passband filtering with the speed of the target, so it is necessary to design MTI filter. The MTI filter used on the radar is generally an FIR filter, because the FIR filter has a linear phase, which is conducive to processing and analysis. Figure 2 illustrates the signal detection process.

Assuming that the radar elevation range is divided into $\mathrm{S}$-wave positions in the elevation dimension, the echo signal is divided into $M$ azimuths in the azimuth dimension, and the echo signal is divided into $\mathrm{N}$ range units in the range dimension, and then, the clutter map has SMN basic unit; the radar clutter data stored in each basic unit are generally the average of all sampled clutter values at different moments in this range and azimuth. Because it is the clutter data at different sampling moments, a stable wave position-distance-azimuth clutter value can be obtained using its own recursive algorithm. In the noise, it is only necessary to output the normal video, which improves the detection performance. The dynamic clutter map scans the surrounding area in real time when the radar is working and updates the clutter value corresponding to the range-azimuth. It contains both fixed clutter and dynamic clutter. Using MTI filtering for the recursively stable clutter storage value can filter out fixed clutter such as ground clutter, and then, the remaining part is the dynamic clutter map, and AMTI processing is performed on the dynamic clutter.

2.4. Sensor Model Weight Recursion. The sensor model needs to compensate the clutter signal spectrum center, move the dynamic clutter spectrum center to zero frequency, and then filter it with an MTI filter, which is equivalent to converting to ground clutter for filtering. Another method is to use a filter with a notch at the center of the clutter spectrum to directly suppress the moving clutter, without moving the center of the clutter spectrum to zero frequency. The filter weight coefficients are stored in a library. This method is often used in engineering. First, we design the MTI filter with the notch at the zero frequency and then shift the frequency axis to obtain the filter weight coefficient library. When the center of the clutter spectrum is estimated, the corresponding filter is directly called just carry out the cancellation process. Incoherent accumulation refers to the addition of the amplitude of $\mathrm{N}$ pulses after the signal passes through the amplitude detector. However, because the detector is nonlinear, the signal cannot be added in phase, so the signal-to-noise ratio cannot reach $\mathrm{N}$ after incoherent accumulation.

To effectively jam the radar, the amplitude of the deception jamming signal is generally higher than that of the real target. The interfered radar received signal is processed by pulse compression. Figure 3 shows the weight distribution of the radar signal sensing model. The result is as shown in the text, and after the pulse compression, the target echo and the interference signal additional time delay have larger pulse pressure peaks, and the radar will not be able to distinguish the true target echo position.

In addition, from the perspective of the circuit, an isolated AC-to-DC circuit is not necessary, and a power supply, namely a non-isolated switching power supply, can also be used. The radar receiver's amplitude gain control (AGC) circuit can reduce the gain. In turn, the radar receiver uses the jamming signal as the real target signal to achieve the purpose of range deception on the radar. The timefrequency distribution of the composite interference signal is mainly the broadband characteristic of suppressed interference, but the bandwidth of the noise amplitude 


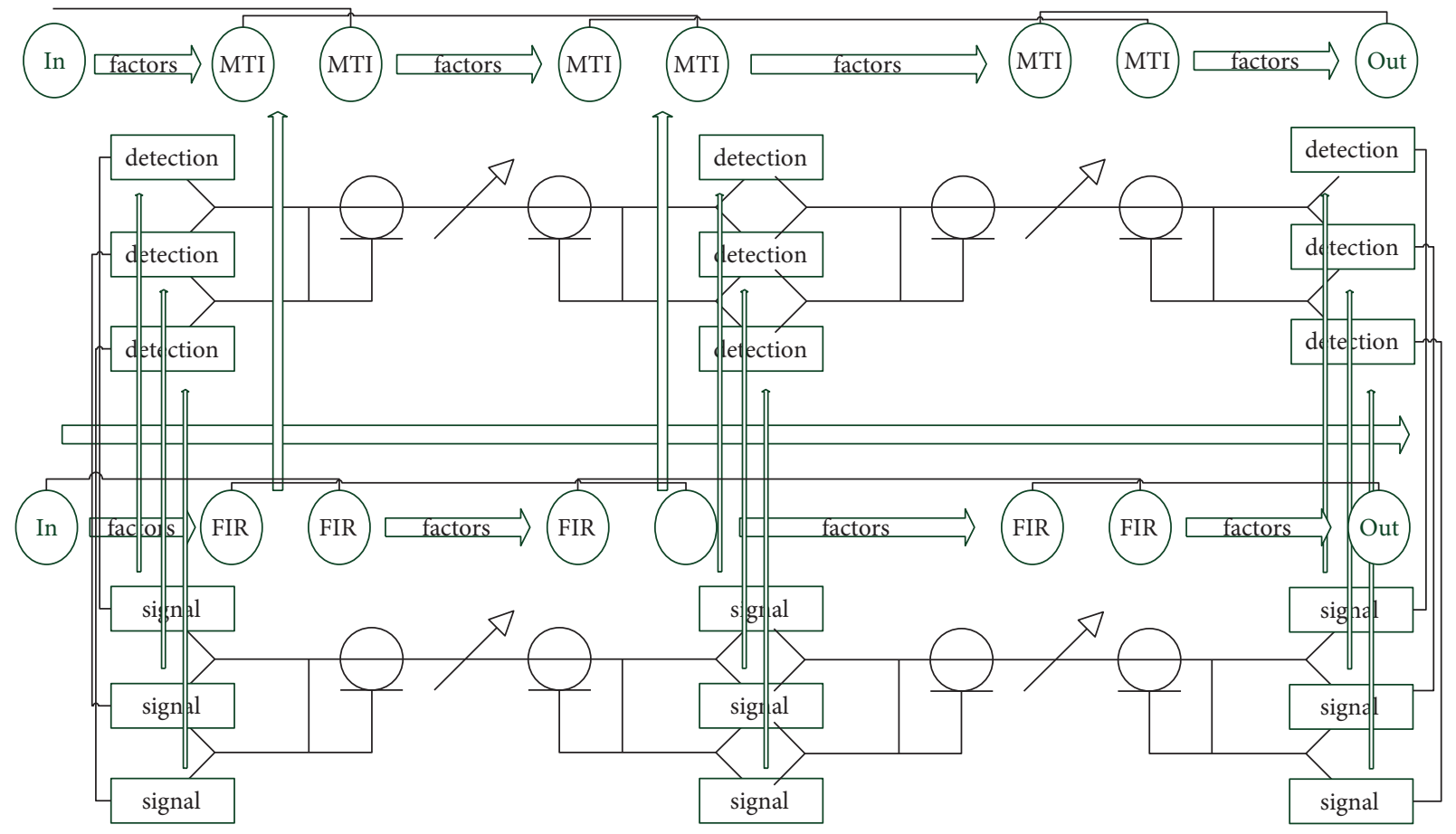

FIgURE 2: Description of signal detection process.

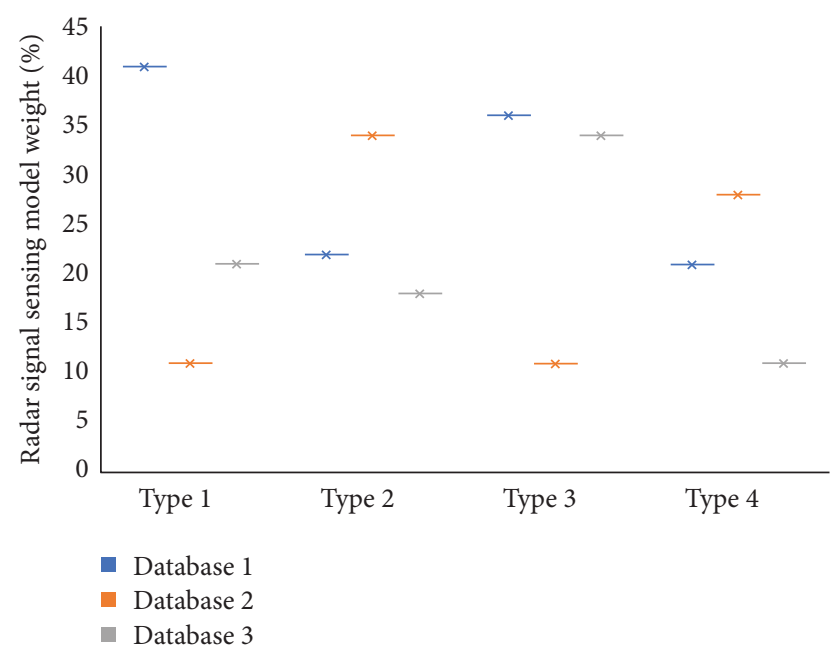

FIGURE 3: Weight distribution of radar signal sensing model.

modulation interference signal is generally not too wide to cover the deceptive interference completely, so it can still be seen in the time-frequency distribution diagram. However, as the composite interference signal itself, the deceptive interference component and the suppressive interference component are both complementary and independent of each other. Therefore, the suppressive interference signal does not have to completely cover the deceptive interference.

\section{Results and Analysis}

3.1. Active Interference Suppression Data Preprocessing. In this signal processing system, the eigenvector method is used to design the filter, and the designed filter coefficients are stored in the external memory. In the design, the center frequency interval of the filter notch is designed to be $5 \mathrm{~Hz}$, and the design range is $0 \mathrm{~Hz}-120 \mathrm{~Hz}$; a total of 25 filters can basically cover all the motion range of the dynamic clutter. When performing AMTI calculation, first the center frequency of the dynamic clutter spectrum is estimated, and then, the filter coefficients are called with the notch at this frequency in the external memory to perform the cancellation operation. The data sending module is used as the sending interface from DSP to FPGA. After completing functions such as clutter suppression, data are sent to this module. The frame header is used to identify the arrival of a frame of data, and the message has multiple attributes of the data, such as working mode, wave position, and pulse code, and then, data are spliced in the erasing mode, and the end of the frame is added after the spliced data as a frame of data. It is judged that the frame data will be sent after the previous frame of data is sent, and the corresponding link port and DMA register are configured. Figure 4 is the echo modulation ratio of the radar jamming signal.

The composite interference signal is the time-domain superposition of noise FM interference and range deception interference. Among them, the modulation factor of noise FM interference signal is 1000. The distance deception jamming delay is $1 \mathrm{us}$, and the target echo signal delay is 5 us. The ratio of noise FM interference component to target echo signal in composite interference is $32 \mathrm{~dB}$, and the ratio of deceptive interference to target echo dSR is set to $35 \mathrm{~dB}$ and $14 \mathrm{~dB}$, respectively. When the JSR is $14 \mathrm{~dB}$, the limit threshold is $-18 \mathrm{~dB}$. It can be seen that when the interference passes through the receiver, the time-domain distribution of blocking interference is more dense than that of aiming interference. In this article, both the blocking jamming 


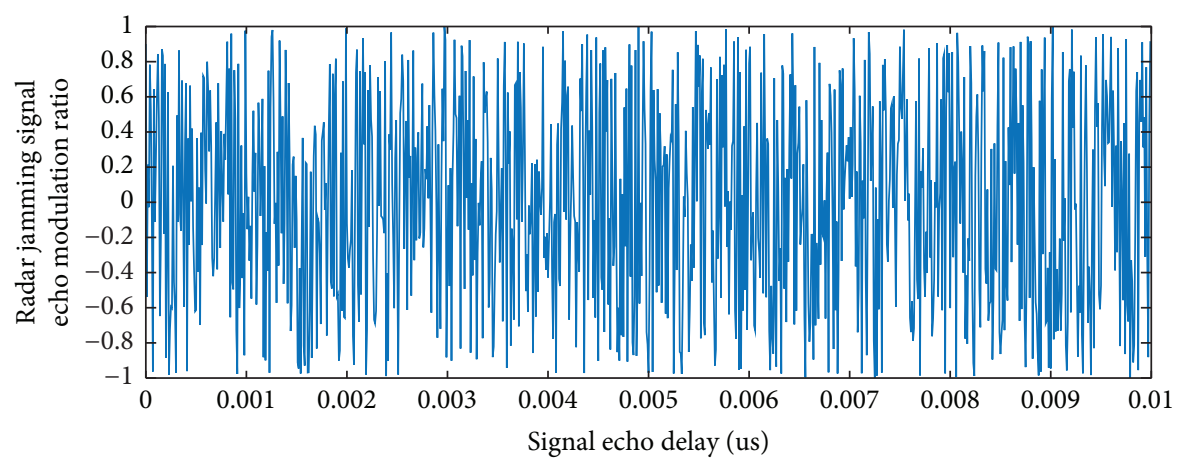

FIgURE 4: Radar jamming signal echo modulation ratio.

bandwidth and the targeting jamming bandwidth are greater than the radar receiver bandwidth. Although the blocking jamming has a larger spectrum range than the targeting jamming, the effective time for the interference to sweep through the radar receiver will be longer than that of the aiming interference. With the increase in SNR, the delay measurement error gradually decreases. When SNR $>-10 \mathrm{~dB}$, the delay measurement error is less than the distance resolution unit $(0.01 \mu \mathrm{s})$, indicating that the distance measurement performance will decrease when the noise level is high. From the algorithm derivation process, it can be seen that this method does not limit the interference type. The algorithm's suppression performance for blocking interference, sweeping interference, and comb spectrum interference is basically the same as that for targeting interference and will not be repeated here.

\section{Simulation of Radar Flaw Detection and Detection Model}

The simulation parameters in this article are set as follows: let the pulse radar transmit a chirp signal with a pulse width of 10 us and a bandwidth of $20 \mathrm{MHz}$, a sampling frequency of $60 \mathrm{MHz}$, a signal-to-interference ratio set to JSR $=10 \mathrm{~dB}$, and a signal-to-noise ratio SNR change range of $0 \mathrm{~dB}$ to $15 \mathrm{~dB}$, and the change interval is $1 \mathrm{~dB}$. In the simulation, the number of SMSP interference sub-pulses is set to $L=4$, the number of C\&I interference sampling signal segments is set to $m=4$, and the number of copying times is $n=5.500$ Monte Carlo tests were carried out under different signal-tonoise ratios, of which 250 groups were training samples and 250 groups were test samples. Due to the special operating mode of the radar used in the field test, although three receiving beams can be formed at the same time corresponding to three receiving channels, the signal forms of the corresponding three transmitting beams are different from each other, making the received signal model in an underdetermined state.

Figure 5 is the echo signal detected by radar flaw detection. It can be seen that the signal-to-interference ratio after the pulse pressure of the recovered target echo signal is more than $40 \mathrm{~dB}$ after the beat and filter processing. In the PCB design, the creepage distance also needs to be considered, and the strong current area and the weak current

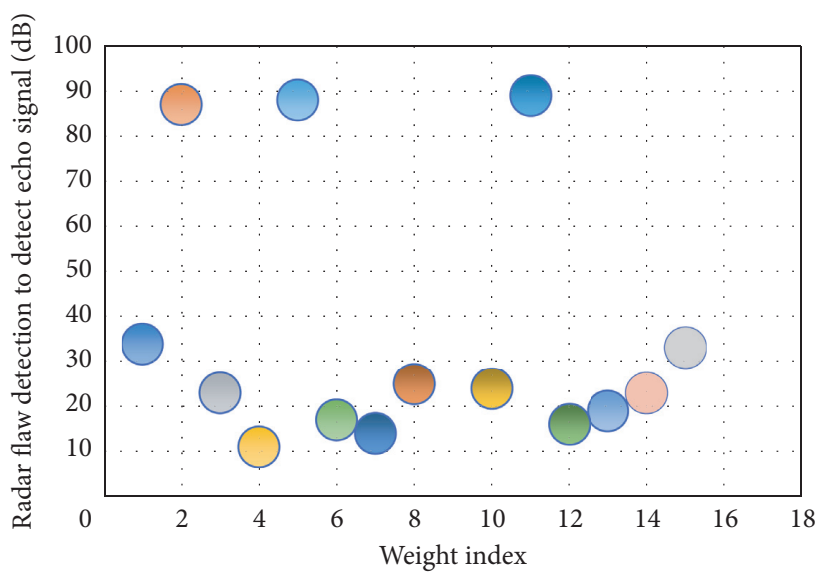

FIGURE 5: Radar flaw detection of echo signal.

area are distinguished by the method of trench isolation. The blind source separation conditions mentioned in this article are not met, so it is difficult to effectively suppress the interference in the measured data using the interference suppression method based on blind source separation. The follow-up research work will gradually develop to address this problem. That is, the post-beat filtering method has a suppression gain of $14+40=54 \mathrm{db}$ for distance false target interference. The time delay of the target echo signal is still 40 us, which means that filtering and noise FM interference cancellation processing do not affect the parameters of the target echo signal. After the interference suppression algorithm is processed, the radar receiver can effectively detect the real target echo signal. It can be seen from the simulation results that the algorithm can improve the radar detection performance by more than $40 \mathrm{~dB}$.

Table 2 shows the frequency sweep interference modulation of the target echo signal. In practice, when the frequency sweep rate is selected for the frequency sweep interference, the interference effect similar to that of the blocking interference can be achieved, resulting in a lower classification and identification probability for these two situations. It can be seen from the above that the probability of correct recognition is above $70 \%$. In the case of pure echo, the amplitude of the echo in the frequency band is generally higher than that of other frequency bands; in the case of targeted interference, the frequency range of the interference 
TABLE 2: Sweep frequency interference modulation of target echo signal.

\begin{tabular}{lccc}
\hline Action number & Signal node & Modulation & Weight frequency/kHz \\
\hline 1 & Hold high & Clenched & 800 \\
2 & Hold high & Right cancellation & 900 \\
3 & Hold high & Scissor cancellation & 1000 \\
4 & Flat pendulum & Left swing & 1100 \\
5 & Flat pendulum & Open sweep & 1200 \\
6 & Flat pendulum & Closed sweep & 1300 \\
\hline
\end{tabular}

is always in the radar receiver work. In the frequency band, the parameter can be used to better separate this situation. It can be seen from the above that the correct identification probability of pure echo and aiming interference is above $85 \%$. This shows that the feature parameters extracted in this chapter are effective, and the identification method based on the decision tree is feasible.

\section{Analysis of Experimental Results}

Experimental simulation conducted a specific analysis of the simulation parameters and simulation data of radio frequency noise interference, including the numerical change in each indicator and the meaning on the corresponding image. The same simulation was performed for noise amplitude modulation, noise frequency modulation, and noise modulation. It has been reserved, and the specific meaning will not be repeated. Now the four interference methods and RPCA denoising processing are compared, and the effect of RPCA denoising is analyzed. It can be seen from the performance curve that the detection probability of the detector changes with the number of training samples, where $\mathrm{SNR}=8 \mathrm{~dB}, N=8$, and $H=4$. The horizontal axis is the number of training samples $K$, and the vertical axis is the detection probability graph and is another important tool for measuring the performance of the detector. Therefore, the relevant data of the original scene image and the low-rank matrix are sorted out; that is, the original image is compared with the denoising process afterwards. When noise interferes, the noise data are reflected in the image with gray value, and the noise distribution is random and the degree of deviation is large, so the mean and variance of the image increase. In the image, due to the presence of noise, the unevenness of the overall image is reflected, and noise is distributed on the original target. The low-rank matrix image decomposed by RPCA is relatively uniform as a whole, and noise can be clearly observed in the sparse matrix image.

Figure 6 shows the distribution of the correlation coefficients of radar jamming signals. It can be found that the instantaneous frequency distribution of SMSP interference and C\&I interference signals is very different. When the number of sub-pulse segments of the C\&I interference signal increase, due to the limitation of the time-frequency resolution capability of the STFT method itself, the time-frequency separation after the SST method is rearranged is poor. The C\&I interference signal in the adjacent gap is a copy of the sampled segment signal, so it has the same frequency distribution as the sampled segment signal. From

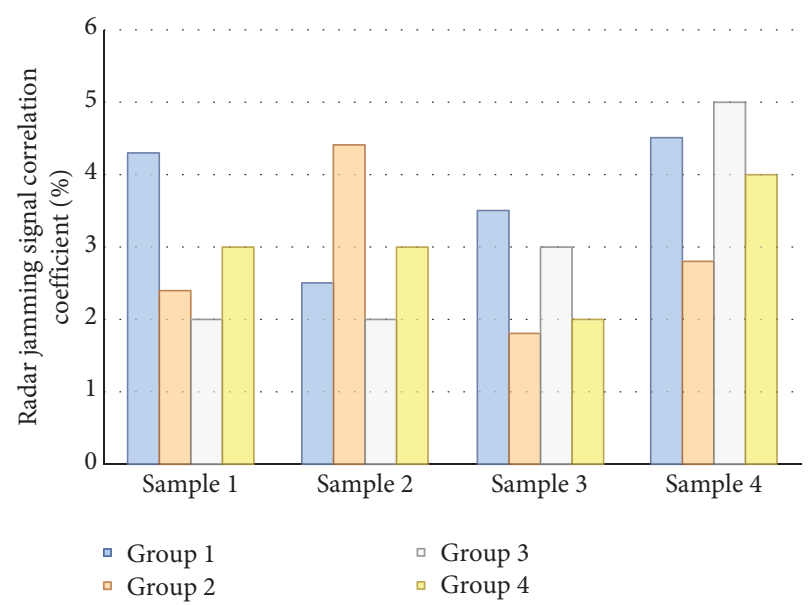

FIgURE 6: Correlation coefficient distribution of radar jamming signal.

the principle of C\&I interference, it can be known that the sampled segment signal has the same frequency modulation slope as the intercepted radar signal.

This power supply simultaneously supplies power to the power metering circuit, central processing unit, relay, and Wi-Fi circuit. Compared with the time-frequency distribution of C\&I signals, the article can no longer accurately give the instantaneous frequency of the signal segment copied in the adjacent gap, and its instantaneous frequency is close to a straight line instead of a small oblique line. Through the comparison of RPCA denoising processing, it can be observed that the relevant parameters of the four interference modes have the same changing trend. The number of zeros has dropped drastically. Among them, the number of iterations of noise amplitude modulation and noise phase modulation is the same and has the least value, and the number of iterations of noise frequency modulation is the largest. After RPCA processes the four interference methods, the ranks of the low-rank matrices are close, which is consistent with the similarity of the correlation coefficients. The images recovered from the low-rank matrices can be observed and confirmed.

Figure 7 is the simulation parameter modulation of radar signal. When the signal-to-noise ratio is low, the recognition rates of the two types of interference under several simulation parameters are relatively good. For example, when the signal-to-noise ratio is $0 \mathrm{~dB}$, the recognition rate of SMSP interference can reach $93.56 \%$, and the recognition rate of C\&I interference is $89.86 \%$, which verifies the effectiveness of the method proposed in this article. It is worth pointing out that because SMSP interference and C\&I interference 


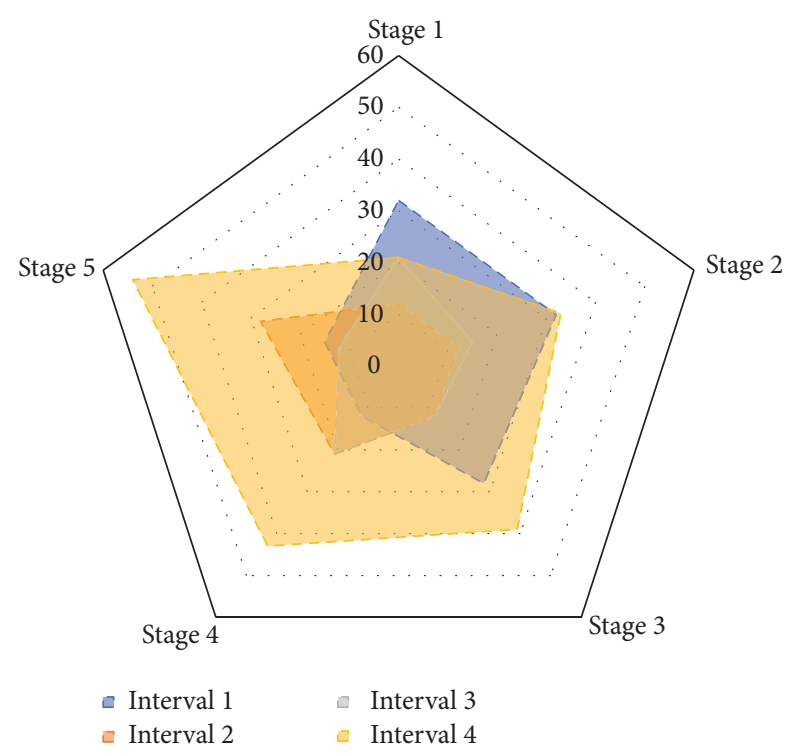

FIgURE 7: Radar signal simulation parameter modulation.

energy need to be large enough to form effective dense false target interference at the radar receiving end, for example, the JSR set in the simulation condition is $10 \mathrm{~dB}$. By comparison, it can be found that the change in interference modulation parameters will affect the identification effect of interference. This is because when the number of interfering signal sub-pulses increases, the SST method cannot accurately separate the instantaneous frequencies of the interfering sub-pulses due to the close distance between the subpulses. This leads to a decrease in the separability of the timefrequency features extracted subsequently, thereby reducing the interference.

\section{Conclusion}

Aiming at the active additive compound interference suppression, this study studies the interference suppression method based on the phase perturbation principle. On the basis of combining the characteristics of the LFM signal, the pulses before and after the radar transmission signal are, respectively, added with approximately orthogonal disturbance phases, and then, the deceptive interference component in the composite interference is strengthened by the matched filter corresponding to the previous pulse repetition period, and then, the target echo signal is recovered by the inverse operation of matched filtering. Because the algorithm is intuitive to suppress compound interference and can use existing conventional interference suppression algorithms, the algorithm has certain practical value. The disadvantage of this algorithm is that the type of composite interference signal needs to be known. In actual use, the algorithm needs to be used in the radar system with the identification of interference types and the selection of antijamming measures. Finally, the validity of the extracted parameters and the feasibility of the identification method are verified through the processing of experimental data. This method extracts characteristic parameters from the time domain and frequency domain, with low computational complexity and good engineering feasibility. This article assumes that the target always exists, so it does not consider only the two situations of noise and noise plus interference. The follow-up research work will start from these two aspects. For the setting of the threshold, human factors are relatively large, and the next step will be to consider the adaptive threshold decision.

\section{Data Availability}

The data used to support the findings of this study are available from the corresponding author upon request.

\section{Conflicts of Interest}

The authors declare that they have no known competing financial interests or personal relationships that could have appeared to influence the work reported in this study.

\section{Acknowledgments}

This work was supported in part by the New Engineering Pilot Project (szxy2018xgk05); Key Disciplines of Computer Science and Technology (2019xjzdxk1); Teaching Research Project (2018jyxm0960); Collaborative Innovation CenterCloud Computing Industry (4199106); and Research Platform Project of Suzhou University (2019ykf30).

\section{References}

[1] S. Li, J. Xie, F. Zhou, W. Liu, and H. Li, "Foreign object intrusion detection on metro track using commodity WiFi devices with the fast phase calibration algorithm," Sensors, vol. 20, no. 12, Article ID 3446, 2020.

[2] B. Ai, A. F. Molisch, M. Rupp, and Z.-D. Zhong, "5G key technologies for smart railways," Proceedings of the IEEE, vol. 108, no. 6, pp. 856-893, 2020.

[3] L. Li and T. -J. Cui, "Information metamaterials-from effective media to real-time information processing systems," Nanophotonics, vol. 8, no. 5, pp. 703-724, 2019.

[4] S. Lee, B. H. Lee, and J. E. Lee, "Statistical characteristic-based road structure recognition in automotive FMCW radar systems," IEEE Transactions on Intelligent Transportation Systems, vol. 20, no. 7, pp. 2418-2429, 2018.

[5] M. A. B. Fayyaz and C. Johnson, "Object detection at level crossing using deep learning," Micromachines, vol. 11, no. 12, Article ID 1055, 2020.

[6] M. Aljohani, A. Mrebit, L. Lo Monte, and M. C. Wicks, "Radar imaging using pseudo-coherent marine radar technology," IET Radar, Sonar \& Navigation, vol. 14, no. 6, pp. 905-916, 2020.

[7] Y.-D. Kim, G.-J. Son, H. Kim, C. Song, and J.-H. Lee, "Smart disaster response in vehicular tunnels: technologies for search and rescue applications," Sustainability, vol. 10, no. 7, Article ID 2509, 2018.

[8] M. Solla, V. Pérez-Gracia, and S. Fontul, "A review of GPR application on transport infrastructures: troubleshooting and best practices," Remote Sensing, vol. 13, no. 4, Article ID 672, 2021.

[9] C. Lin, X. Wang, Y. Li, F. Zhang, Z. Xu, and Y. Du, "Forward modelling and GPR imaging in leakage detection and 
grouting evaluation in tunnel lining," KSCE Journal of Civil Engineering, vol. 24, no. 1, pp. 278-294, 2020.

[10] E. Yurtsever, J. Lambert, A. Carballo, and K. Takeda, "A survey of autonomous driving: common practices and emerging technologies," IEEE access, vol. 8, pp. 58443-58469, 2020.

[11] M. M. Bahador, A. Zaimbashi, and R. Rahgozar, "Three-stage Lamb-wave-based damage localization algorithm in plate-like structures for structural health monitoring applications," Signal Processing, vol. 168, Article ID 107360, 2020.

[12] W. Feng, J. M. Friedt, and S. Wang, "Passive bistatic radar using digital terrestrial television broadcasting signal for subsurface target detection," in Proceedings of the 2019 Photonics \& Electromagnetics Research Symposium - Fall (PIERS - Fall), pp. 586-597, Xiamen, China, December 2019.

[13] I. Orr, M. Cohen, and Z. Zalevsky, "High-resolution radar road segmentation using weakly supervised learning," Nature Machine Intelligence, vol. 3, no. 3, pp. 239-246, 2021.

[14] P. Chiariotti, M. Martarelli, and P. Castellini, "Acoustic beamforming for noise source localization - reviews, methodology and applications," Mechanical Systems and Signal Processing, vol. 120, pp. 422-448, 2019.

[15] J. Fayyad, M. A. Jaradat, D. Gruyer, and H. Najjaran, “Deep learning sensor fusion for autonomous vehicle perception and localization: a review," Sensors, vol. 20, no. 15, Article ID 4220, 2020.

[16] S. R. Zekavat, R. M. Buehrer, and G. D. Durgin, "An overview on position location: past, present, future," International Journal of Wireless Information Networks, vol. 28, pp. 18-32, 2021.

[17] C. M. Furse, M. Kafal, and R. Razzaghi, "Fault diagnosis for electrical systems and power networks: a review," IEEE Sensors Journal, vol. 21, no. 2, pp. 888-906, 2020.

[18] Y. Xue, F. Kong, S. Li et al., "Water and mud inrush hazard in underground engineering: genesis, evolution and prevention," Tunnelling and Underground Space Technology, vol. 114, Article ID 103987, 2021.

[19] Z. Wang, Y. Wu, and Q. Niu, "Multi-sensor fusion in automated driving: a survey," IEEE Access, vol. 8, pp. 2847-2868, 2019.

[20] S. Liu, L. Li, and J. Tang, "Creating autonomous vehicle systems," Synthesis Lectures on Computer Science, vol. 6, no. 1, Article ID 186, 2017.

[21] S. Li and J. Li, "Condition monitoring and diagnosis of power equipment: review and prospective," High Voltage, vol. 2, no. 2, pp. 82-91, 2017.

[22] Y. Liu, Y. Ye, Q. Wang, and W. Wang, "Experimental research on shear failure monitoring of composite rocks using piezoelectric active sensing approach," Sensors, vol. 20, no. 5, Article ID 1376, 2020.

[23] Y. Cheng, J. Su, and H. Chen, "A new automotive radar 4d point clouds detector by using deep learning," in Proceedings of the ICASSP 2021 - 2021 IEEE International Conference on Acoustics, Speech and Signal Processing (ICASSP), pp. 83988402, Toronto, ON, Canada, June 2021.

[24] Y. Fujino, D. M. Siringoringo, Y. Ikeda, T. Nagayama, and T. Mizutani, "Research and implementations of structural monitoring for bridges and buildings in Japan," Engineering, vol. 5, no. 6, pp. 1093-1119, 2019. 